\title{
IN MEMORIAM RAMÓN MARTÍN MATEO
}

\author{
JAVIER SERRANO
}

Abogado

Vicepresidente de ADAME

El pasado 22 de mayo falleció en San Juan (Alicante) mi querido amigo y maestro D. Ramón Martín Mateo. En estos días los medios de comunicación y, en especial, la comunidad académica ha glosado la figura del maestro en su amplia proyección social, subrayando la imborrable huella de su personalidad y su quehacer, tanto desde la perspectiva como gestor universitario, su compromiso social con la Universidad y la enseñanza del derecho, la investigación, los avances científicos y tecnológicos y los problemas de nuestro entorno.

Autor de más de cuarenta libros y trescientos artículos doctrinales, participa en diversos proyectos nacionales e internacionales. Experto de Naciones Unidas, vocal electo del Consejo de Estado, presidió numerosas instituciones e impulsó diversas revistas especializadas. Doctor honoris causa por las Universidades de León, Mendoza, País Vasco, Rovira i Virgili y Murcia. Recibió diversas distinciones, entre las que destacan la Orden del Mérito Civil; el Premio Nacional de Medio Ambiente (1992); el Premio Rey Jaume I de la Generalitat Valenciana a la protección del medio ambiente; y el Premio Justicia de la Generalitat Valenciana (2001).

Ramón Martín Mateo nace en Villabrágima (Valladolid), el 31 de agosto de 1928. Después de una primera etapa profesional que desarrolla como miembro del Cuerpo de Interventores de Administración Local, llega al ámbito universitario de la mano del profesor García de Enterría, desplazándose a la Universidad de Múnich para redactar su tesis doctoral sobre el Municipio y el Estado en Derecho en Alemania, para posteriormente incorporarse a la Universidad en su primera cátedra de Derecho Administrativo en la Facultad de Económicas de Bilbao, en 1968.

Secretario General y Rector de la Universidad del País Vasco (1976-1979). En 1982 se incorpora a la Universidad de Alicante como Catedrático de Derecho Administrativo. En esta Universidad desempeñó el cargo de Rector de 1986 a 1994, siendo su primer 
Rector estatutario de esta última, impulsando la configuración y desarrollo de su actual campus universitario.

Estudioso de la Administración Local y de sus formas de organización, fue un convencido municipalista y defensor de los medios que precisan para el ejercicio de sus competencias. En la década de los años setenta, y desde la experiencia profesional en la administración local, comienza a abordar en sus estudios y artículos doctrinales los problemas propios del medio ambiente urbano. Sirva a título de ejemplo sus artículos sobre la administración de la atmósfera (REDA 1976) o la planificación ambiental en la reforma de la ley del suelo (Revista de Derecho Urbanístico 1976), a los que seguirían numerosos más durante los ochenta y noventa. Al mismo tiempo va conformando los pilares de lo que sería la publicación del primer tratado de Derecho ambiental en nuestro país, que vio la luz en 1977 publicado por la editorial IEAL.

Promulgada la Constitución Española y tras nuestra incorporación a la Unión Europea en 1985, en 1991 aparece el primer volumen del Tratado de Derecho Ambiental, una actualización del tratado de 1977, primero de los cuatro volúmenes publicados por la editorial Trivium, su principal obra en el campo del derecho ambiental. Esta obra supone la primera construcción doctrinal y sistematización de los principios básicos del derecho ambiental que van a inspirar el trabajo de numerosos iusambientalistas en las siguientes décadas, tanto en España como en Iberoamérica.

El volumen I del Tratado recoge la nueva configuración constitucional del ambiente, el reparto competencial entre las distintas administraciones y lo que denomina los ordenamientos concluyentes; el papel de las nuevas técnicas reguladoras; la evaluación de impacto ambiental, los derechos de acceso a la información y participación pública; las nuevas técnicas de intervención; la planificación y los instrumentos de mercado, así como el derecho de la Unión Europea e internacional y el impulso que a las técnicas reguladoras supuso. El tratado lo completará con los volúmenes II (1992), dedicado a los aspectos sectoriales y III (1997) a los recursos naturales, este último con la colaboración de Juan José Díaz Sánchez, catedrático de Derecho Administrativo de la Universidad de Alicante.

En la obra de Ramón Martín Mateo es constante la preocupación por la relación entre las ciencias de la naturaleza y las ciencias sociales, que entiende enormemente distanciadas de los avances científicos y tecnológicos, y a lo que dedica especial 
atención en su obra El hombre: una especie en peligro (Campomanes Libros, Madrid 1993).

$\mathrm{Su}$ intensa inquietud intelectual le lleva a interesarse vivamente por los progresos científicos y los retos que les plantean a las ciencias sociales, en especial al Derecho, reflexionando sobre la técnicas regulatorias y la interdisciplinariedad de los conocimientos, con el fin de que el derecho se convierta en un instrumento eficaz para el progreso humano y la preservación de nuestro entorno. Todo ello en consonancia con su propia definición como ingeniero social, eso sí, buenhumorado, tal y como nos relata en su libro del mismo título.

Tomando sus propias palabras: El Derecho no es ni un saber abstracto ni una técnica cerrada al servicio de su propio progreso. Parte de la realidad social, íntimamente vinculada en nuestro caso con los fenómenos naturales, que se intenta mejorar y ordenar positivamente. De aquí que sea imprescindible el conocimiento de los mecanismos en los que van incidir las leyes para entender y valorar, en términos de eficacia, su contenido. Hay, pues, un previo ejercicio de pedagogía extrajurídica que se intenta en la obra, pero también, a la inversa, se asume el propósito de facilitar la asimilación de las técnicas jurídicas por otros profesionales y expertos, partiendo de la poco discutible premisa de que el Derecho es para todos y no un coto cerrado de determinados especialistas.

Especial relevancia otorga en su obra a las energías renovables, que defenderá a lo largo de su dilatada carrera. También en la década de los años setenta y comienzo de los ochenta les dedica especial atención: Planificación Solar (Revista de Derecho Urbanístico, Madrid Enero de 1983); Presupuestos de la regulación administrativa en el campo energético (Homenaje a José Antonio García Trevijano, IEAL. Madrid 1982) o su obra Nuevo Derecho de la Energía (Instituto de Estudios de la Administración Local. Madrid 1983), entre otras.

Ramón Martín Mateo fue pionero en la formación y divulgación del Derecho ambiental, impartiendo a partir de 1989 los primeros cursos de doctorado en derecho ambiental de nuestro país en el Departamento de Derecho Administrativo de la Facultad de Derecho de la Universidad de Alicante, que atendiendo a su vocación internacional en 1993, se ampliaron a licenciados iberoamericanos. Desde entonces han sido numerosos los profesionales del Derecho que han obtenido su formación bajo la tutela de Ramón Martín Mateo, cuya figura cobra especial trascendencia en toda Latinoamérica. 
En 1989 funda, junto con otros profesores de su departamento, la Asociación de Derecho Ambiental Español (ADAME), que en estas fechas cumple veinticinco años de existencia. Durante esta etapa han sido diversos los proyectos que bajo el impulso de la Asociación se han desarrollado, destacando la organización de los Congresos Nacionales de Derecho Ambiental y la elaboración de la Revista Thomson-Aranzadi de Derecho Ambiental, que recientemente ha cumplido diez años.

Tuve la fortuna de conocer al profesor Martín Mateo en 1993, cuando fui a exponerle, junto con otros profesionales del Derecho, la intención de organizar un congreso nacional de derecho ambiental, iniciativa que, en su primera edición, en 1995 en Sevilla contaba con el apoyo del profesor Luciano Parejo y con la organización de mi despacho profesional, así como con la Fundación de la Universidad Carlos III de Madrid, solicitándole que la Asociación conformara el Comité Científico. ADAME tomó el relevo en las ediciones posteriores, celebrándose con carácter bianual hasta en ocho ocasiones.

Los congresos respondían a su propia filosofía, pretendiendo aunar en el debate a académicos; funcionarios; fiscales; magistrados y profesionales del derecho y de otras disciplinas, en mesas de trabajo en las que se han sucedido durante estos quince años centenares de ponentes. Bajo su dirección y tutela hemos sido testigos de la evolución del derecho ambiental en nuestro país, impulsando con esa orientación de ingeniero social buenhumorado.

Singular entre la variada y brillante doctrina científica española, sin duda su impulso ha contribuido de manera notable a la formación de muchos ambientalistas, al desarrollo de jóvenes juristas que en las últimas décadas han dado cuerpo a una generación de brillantes especialistas en nuestro país, que en la actualidad desarrollan su labor tanto en el ámbito universitario como en el profesional.

Ramón Martín Mateo era ante todo un humanista, vivía con pasión su vocación más allá del ámbito universitario. Trabajador incansable, a quien siempre acompañaba una libreta en la que anotaba continuamente sus observaciones, se mantuvo siempre inmensamente activo en la Universidad como profesor emérito más allá de su temprana jubilación. Innovador y predictivo, bien lo saben sus discípulos. Combinaba el rigor académico con la permanente observación de lo que acontecía en su entorno, con análisis provistos de fina ironía y un excelente buen humor. Excelente conversador, columnista e incluso inventor, observador de la cotidianeidad en sus colaboraciones 
periódicas en el diario Información de Alicante y La Verdad de Murcia, recopiladas algunas de ellas en el entrañable libro Lecturas para el desayuno.

De inmensa talla intelectual, heterodoxo y comprometido. Provisto de una notable intuición para adelantarse a su tiempo, y un tanto incómodo para el establishment, sus intervenciones públicas siempre podían tener una deriva insospechada. Algunos todavía recordamos su intervención en la clausura del Congreso de Valencia en 1997, en la que arremetió contra el papel que estaban desempeñando la mayoría de las cajas de ahorro, aperitivo de lo que sucedería diez años después con el estallido de la crisis financiera.

Ramón nunca dejó de denunciar la necesidad de preservar nuestro medio y el deber intergeneracional desde una perspectiva global y universalizadora, sin abandonarse como él bien decía, al Muro de las Lamentaciones, proponiendo soluciones siempre innovadoras.

En él siempre encontramos la atención y orientación necesaria, las puertas de su despacho siempre estuvieron abiertas para todos, en especial para los profesionales ajenos al mundo universitario a quienes siempre acogió y con quienes compartió experiencias y conocimiento. Querido Ramón, nos hemos quedado sin libreta. 